\title{
Cytokeratins in gastroenterology. Systematic review
}

\author{
Marek Pastuszak ${ }^{1}$, Krzysztof Groszewski ${ }^{1}$, Małgorzata Pastuszak², Przemysław Dyrla ${ }^{3}$, Stanisław Wojtuñ ${ }^{3}$, Jerzy Gil ${ }^{3}$ \\ ${ }^{1}$ Department of Gastrointestinal Endoscopy, Provincial Hospital, Elblag, Poland \\ ${ }^{2}$ Department of Internal Diseases, Center of Internal Diseases ElVita, Elblag, Poland \\ ${ }^{3}$ Department of Gastroenterology, Military Institute of Medicine, Warsaw, Poland
}

Prz Gastroenterol 2015; 10 (2): 61-70

DOI: $10.5114 /$ pg.2015.51182

Key words: keratin, gastroenterology, pathophysiology, systematic review.

Address for correspondence: Marek Pastuszak MD, Department of Gastrointestinal Endoscopy, Provincial Hospital, 146 Królewiecka St, 82-300 Elblag, Poland, phone: +48 603062 918, e-mail: marcuswp@wp.pl

\begin{abstract}
Keratins are proteins that form intermediate filaments of epithelial cell cytoskeleton. The utility of keratin expression determination is based on the fact that epithelial cells acquire a specific pattern of keratin expression during differentiation and maturation, which reflects the specificity of the tissue and the degree of maturation, and generally remains stable during carcinogenesis. Determination of the pattern makes it possible to identify the origin of cells in diagnosing neoplastic lesions as well as in research on pathophysiology or the possibility to apply keratin-positive cell detection in the process of cancer staging and treatment planning. As keratins undergo degradation during apoptosis as caspase substrate the identification of the caspase-derived K18 fragment by the use of specific monoclonal antibody allows us to estimate the apoptosis/necrosis ratio, especially in liver pathology, e.g. nonalcoholic steatohepatitis, chronic hepatitis or graft-versus-host disease or in assessing response to antiviral or antitumour therapy.
\end{abstract}

Internal organisation of the eukaryotic cells is based on a three-dimensional structure of the cytoskeleton, which affects the important life functions of the cell by its dynamic changes. The cytoskeleton is built up from three main groups of filament-forming proteins, namely: microtubules, microfilaments, and intermediate filaments.

Microtubules, $23 \mathrm{~nm}$ in diameter, are composed of tubulin ( $\alpha$ and $\beta$ ) heterodimers, which form a structure of 13 parallel protofilaments. Within the cells microtubules stretch from the centrosome towards the periphery, forming the cell's basic skeleton, responsible for the intracellular transport processes (including the formation of the mitotic spindle). Microfilaments, with a diameter of $6 \mathrm{~nm}$, are built of the actin mesh in the form of interlaced strands (F actin). Microfilaments are responsible for the maintenance of the cell's shape and they provide resistance to stress/mechanical injury. Microfilaments also participate in the interaction between the cell and its environment - including both the neighbouring cells and the matrix. Intermediate filaments, with a diameter of about $10 \mathrm{~nm}$, are the third element of the cytoskeleton. There are six types of intermediate filaments: the first four-intracytoplasmatic type $\mathrm{V}$ filaments - are associated with the cell nucleus, and type $\mathrm{VI}$ are found in the eyeball. Types I and II are keratins - intermediate filaments of the epithelial cells. Type III includes desmin of the muscle cells, vimentin found in mesenchymal cells, fibroblasts, lymphocytes and endothelial cells, peripherin from neurons, and glial fibrillar acidic protein (GFAP). Type IV intermediate filaments are found mainly in the nerve cells (axons) and in the muscle cells. Type $\mathrm{V}$ are lamins - proteins associated with the nucleus (lamins are considered to be a group of the most conservative, and phylogenetically the oldest, of all intermediate filaments). Type VI includes phakinin and filensin - eye lens protein (now designated as Bfsp1 and Bfsp2).

The keratin group is defined as the group of intermediate filaments of the epithelial cells.

Keratins, forming particles from $44 \mathrm{kDa}$ to about $66 \mathrm{kDa}$ molecular weight, are characterised by high stability and chemical resistance. Keratins have a complex spatial structure, with a highly conserved central core domain, built of an average of 310 amino acid residues (range: 300 to 330) forming the $\alpha$-helical shape limited by the two terminal domains ( $\mathrm{N}$-terminal - head; and C-terminal - tail), which do not form helices and are of 
variable length. The head domain comprises two subdomains, coded $\mathrm{V} 1$ and $\mathrm{H} 1$. The tail domain is formed by two other subdomains - V2 and $\mathrm{H} 2$. Within the core domain four subdomains (forming coils) were described $-1 \mathrm{~A}, 1 \mathrm{~B}, 2 \mathrm{~A}$, and $2 \mathrm{~B}$, connected by L1, L12, and L2 linker sequences. Heteropolimerisation is a characteristic feature of keratins. Keratins form filamentary structures by connecting two keratin molecules - one of type I and one of type II (an $\alpha$-helical structure is used to form an intercoiled structure within the core domains). The intercoiling allows the formation of heterodimers, and heterotetramers, which are the basic units of the keratin filamentous structure. Unlike microtubules or microfilaments, intermediate filaments do not form uniformly polarised structures. Dimers bind together in an anti-parallel manner to form tetramers. Keratin polymerisation is spontaneous and does not require any additional factors. The polymerisation/depolymerisation process is regulated by protein phosphorylation, which affects the properties of keratins, including solubility, spatial structure, and the structure of the forming filaments. The post-translational modifications are also influenced by phosphorylation (which modifies interactions with associated proteins and thereby affects the communication process). Under normal conditions, keratins not involved in heterodimers undergo quick degradation. A total of 54 genes are described in the keratin gene family, making it one of the largest gene families in human biology [1].

Within the epithelial cells keratin filaments form spatial bunches (tonofilaments). Tonofilaments surround the cell nucleus in the cytoplasm, they connect with desmosomes and hemidesmosomes of the cellular membranes that regulate the cohesion, shape, and mechanical resistance and participate in many other functions, including proliferation control, apoptosis, intracellular transport, function of the cellular organelles and their spatial organisation within the cell, and stress response, as well as in malignant transformation. Tonofilaments also play an important role in preserving the integrity of the epithelial tissue by participating in the intercellular interactions between the epithelial cells, as well as between the cells and the basal membrane or surrounding matrix [2].

Keratin studies started with hair biology research initially they emerged during the investigation of wool in the 1970s. Attention was drawn to keratins in human pathology by the discovery that mammalian cells show specific keratin expression patterns depending on the origin of the tissue, and on the cell differentiation and maturation status. In 1982 the first "keratin catalogue" was published - initially containing 19 entries for different human keratins (Moll et al.). This catalogue, based on dividing keratins into two types (type I - "acidic" keratins and type II - "basic and neutral" keratins), despite being initially intended only as a proposal of a provisional classification, is nowadays, after augmentation with recent discoveries, especially regarding new keratins involved in the formation of the skin appendages, still the basis of the recommended classification (as proposed by Schweizer et al. in 2006 and in concordance with HUGO (Human Genome Organisation) nomenclature on unification of the keratin coding genes nomenclature).

The keratin catalogue, currently in operation, distinguishes two main groups: type I, comprising 28 keratins (epithelial keratins: CK9-10; CK12-20; CK23-24; epithelial keratins related to the hair follicle: CK25-28; hair keratins: CK31-40 (including CK33a and CK33b) - 17 genes for epithelial keratins and 11 for hair keratins) and 26 type II keratins (epithelial keratins: CK1-8 (including CK6a, CK6b, and CK6c); CK76-80; epithelial keratins related to the hair follicle: CK71-75; hair keratins: CK8186 - totalling 20 genes for epithelial keratins and 6 for hair keratins). Genes encoding keratins are localised in two main loci of the human genome: chromosome $17 q 21.2$ is a locus for type I keratins with the exclusion of CK18, while chromosome 12q13.13 holds a locus encoding type II keratins and CK18 [1]. For practical purposes a group of "simple" cytokeratins is distinguished (typical for simple and glandular epithelia and for the specialised cells of the mesenchymal organs: CK7, CK8, CK18, CK19, CK20) and "complex" cytokeratins (found in striatified epithelia: CK5/6, CK10, CK14, CK15) (Table I).

Table I. The classification of keratins

\begin{tabular}{lrr}
\hline Keratins & Type 1 & Type 2 \\
\hline Epithelial cells & CK9-10; CK12-20; CK23-24 & CK1-8 (including CK6a; CK6b; CK6c); CK76-80 \\
\hline "Simple" & CK18; CK19; CK20 & CK7; CK8 \\
\hline "Complex" & CK10; CK14; CK15 & CK71-75 \\
\hline Related to the hair follicle & CK25-28 & CK81-86
\end{tabular}


Table II. Cytokeratins in gastroenterology - examples

\begin{tabular}{|c|c|c|c|c|}
\hline \multicolumn{5}{|c|}{ The use of the determination of cytokeratins in gastroenterology - examples } \\
\hline \multicolumn{5}{|c|}{ Identification of the pattern of expression } \\
\hline \multirow[t]{2}{*}{ Diagnosis of cancer } & \multirow{2}{*}{$\begin{array}{l}\text { Research on the } \\
\text { pathophysiology of diseases }\end{array}$} & \multicolumn{3}{|c|}{ Identification of $\mathrm{CK}+$ cells } \\
\hline & & Staging & Prognosis & Basic research \\
\hline \multicolumn{5}{|c|}{ Determination of soluble fragments (assessment of apoptosis/necrosis) } \\
\hline \multirow{2}{*}{$\begin{array}{c}\text { Liver pathology } \\
\text { (e.g. NASH, viral hepatitis) }\end{array}$} & \multirow[t]{2}{*}{ Graft rejection } & \multicolumn{3}{|c|}{ Response to the treatment } \\
\hline & & Neop & & l treatment \\
\hline
\end{tabular}

The significance of keratin defects is best recognised in dermatological pathologies. Research indicates the presence of more severe symptoms when point mutations change the structure (and affect the functions) of individual keratins in comparison to the defects leading to a decreased concentration or lack of a particular keratin. This can be explained by the ability to replace missing keratins in heterodimers by another keratin of a similar function. Regularly updated information is available online at www.interfil.org (Human Intermediate Filament Database). It is important to note that the role of keratins in pathology is not purely mechanical. New data point to keratins being involved in trophoblast pathologies and in the formation of the placenta. They participate in the protection of the cells against injuries (this function is especially recognised in regard to hepatocytes). Keratins seem to have an antiapoptotic function, regulate protein synthesis, and play a role in wound healing as well as in the polarisation of epithelial cells and signal transduction - both intra- and intercellular.

Since the 1980s keratins have also been recognised in the diagnostics and treatment of cancer. Epithelial cells express a specific and changing pattern of cytokeratin expression upon development and differentiation. This pattern is only slightly, if at all, affected by the neoplastic transformation, which allows identification of the cancer cell origin and the stage of tissue differentiation. This can be of particular importance in the case of metastatic cancer of unknown primary site as well as in cases of poorly differentiated, disseminated, progressed cancer involving multiple organs. Identification of cytokeratins in the bone marrow and in the peripheral blood can serve as a measure of circulating cancer cells, which can be used in assessing the clinical course of cancer and in prognosis. Measurement of soluble cytokeratin fragments can served as a measure of the treatment response (Table II).

Two types of tests are used in clinical practice: nonspecific - recognising fragments of native keratins and tests identifying epitops generated during the apopto- sis process due to kaspase digestion of keratins. The first group of tests include the most commonly used tests against TPA (tissue polypeptide antigen - against CK8, CK18, and CK19), TPS (tissue polypeptide specific antigen - against CK18), and CYFRA 21-1 (against CK19 fragment). The second group of tests is based on the concept that in the process of apoptosis keratins are a substrate for caspases. CK18 is of special importance in this regard because, as well as a common region of type I keratins, it possesses a specific site for the caspase 3 action (Asp396), which cleaves an epitope fragment recognised by the M30 antibody (Table III).

In the case of epithelial tumours adenocarcinomas usually present the biggest problem. The fact that the majority of glandular epithelial cells and mesenchymal organs express keratins CK8, CK18, and CK19 (except for hepatocytes, follicular pancreatic cells, renal proximal tubular cells, and cells in some endocrine organs), while the expression of CK7 and CK2O is much more diverse, is used in the diagnostics of adenocarcinomas. Classic diagnostic systems are based, in the first phase, on the qualitative and quantitative evaluation of CK7 and CK20 phenotypes of the cancer cells, which can be further worked up to a wider panel based on the initial results. In case of poorly differentiated neoplasms, the detection of keratins speaks strongly in favour of their epithelial origin. Only in rare instances can low CK8 and CK18/19 expression be found in smooth muscle cells, fibroblasts, lymph nodes, and in some leiomyosarcomas (where they are found along with vimentin and desmin). The keratin profile changes upon the differentiation and maturation of the cells - mature hepatocytes, highly specialised and polarised cells, show only the expression of CK8/18, while foetal hepatoblasts express CK7, CK14, and CK19. A research project investigating keratin expression in 435 cancer cases showed CK7 expression in most carcinomas with the exclusion of those originating from the colon, prostate gland, kidney, thyroid, neuroendocrine tumours of the GI tract, and in Merkel cell carcinoma of the skin, no CK7 expression was found in squamous tumours, with the exception of the cer- 
Table III. Cytokeratins in gastroenterology - possible clinical applications

\begin{tabular}{|c|c|}
\hline CK & Possible applications \\
\hline & Determination of "native" keratins \\
\hline CK7/CK20 & $\begin{array}{l}\text { Identification of cancer of unknown origin } \\
\text { CK7(+)/CK2O(-): prognostic value in colon cancer }\end{array}$ \\
\hline CK8/CK18 & $\begin{array}{l}\text { Presence of mutation: increase of risk of end-stage liver disease } \\
\text { CK18(+) in the bone marrow after surgery: prognostic value in colon cancer }\end{array}$ \\
\hline CK19 & $\begin{array}{l}\text { Distinguishing hepatocellular carcinoma from cholangiocarcinoma and metastatic tumours } \\
\text { Prognostic value in cholangiocarcinoma }\end{array}$ \\
\hline CK10/CK19 & Prognostic value in hepatocellular carcinoma \\
\hline CK17 & In poorly differentiated adenocarcinoma, suggests the origin of the pancreatic ducts \\
\hline CK20/COX-2 & Differences in expression in cancer of left and right part of colon \\
\hline CK7/MUC-6 & The potential role in the distinguishing sporadic from ulcerative colitis-associated colon cancer \\
\hline \multicolumn{2}{|c|}{ Determination of caspase-cleaved fragments of keratins (CK18 - caspase 3 - antibodies M30 and M65) } \\
\hline \multicolumn{2}{|c|}{ Evaluation of the effects of chemotherapy (colorectal, breast, bronchial, and prostatic gland cancer) } \\
\hline \multicolumn{2}{|c|}{ Prognostic value in colon cancer } \\
\hline \multicolumn{2}{|c|}{ Distinguishing NASH from simple steatosis } \\
\hline \multicolumn{2}{|c|}{ Potential role in differentiating inactive HBV carriers from patients with chronic hepatitis B with $\mathrm{HBeAg}(-)$} \\
\hline Correlation to & iviral therapy (HBV) \\
\hline
\end{tabular}

Table IV. Cytokeratins in neoplasms of the gut (based on NCCN Clinical Practice Guidelines in Oncology)

\begin{tabular}{|c|c|c|c|c|}
\hline CK2O & CK7(+) & & CK7(-) & \\
\hline \multirow[t]{2}{*}{ CK2O(+) } & $\begin{array}{c}\text { Pancreas } \\
\text { Cholangiocarcinoma } \\
\text { Stomach }\end{array}$ & $\begin{array}{l}65 \% \\
65 \% \\
40 \%\end{array}$ & $\begin{array}{l}\text { Colon } \\
\text { Stomach }\end{array}$ & $\begin{array}{l}80 \% \\
35 \%\end{array}$ \\
\hline & $\begin{array}{c}\text { Exclusion: } \\
\text { - Neuroendocrine tumour } \\
- \text { HCC } \\
\text { - Oesophagus - squamous }\end{array}$ & & $\begin{array}{c}\text { Exclusion: } \\
\text { - Cholangiocarcinoma } \\
- \text { HCC } \\
\text { - Oesophagus - squamous } \\
\text { - Pancreas }\end{array}$ & \\
\hline \multirow[t]{2}{*}{ CK2O(-) } & $\begin{array}{c}\text { Pancreas } \\
\text { Cholangiocarcinoma }\end{array}$ & $\begin{array}{l}30 \% \\
30 \%\end{array}$ & $\begin{array}{c}\text { HCC } \\
\text { Neuroendocrine tumour (intestine) } \\
\text { Oesophagus - squamous }\end{array}$ & $\begin{array}{l}80 \% \\
80 \% \\
70 \%\end{array}$ \\
\hline & $\begin{array}{l}\text { Exclusion: } \\
\text { Colon }\end{array}$ & & $\begin{array}{c}\text { Exclusion: } \\
\text { - Cholangiocarcinoma } \\
\text { - Pancreas }\end{array}$ & \\
\hline
\end{tabular}

vix. CK20 expression was found in almost all colorectal cancers and in Merkel cell skin tumours, in $62 \%$ of cases of the pancreatic cancer, $50 \%$ of gastric tumours, $43 \%$ of cholangiocarcinomas, and in $29 \%$ of transitional epithelium cancers. No expression of CK20 was found in most other cancers or in mesothelioma. A negative expression profile of $\mathrm{CK} 7 / 20$ was characteristic for the hepatocellular carcinoma (HCC), prostate cancer, and renal clear cell carcinoma (RCC) [3] (Table IV).
Keratin typing is of the utmost importance in colon cancer. Typical cases show strong expression of CK20, with no, or only weak (in comparison to CK20), expression of CK7. Determination of the keratin expression profile in colon cancer is not only important for identification purposes. Studies on colorectal cancer show a correlation between CK20/7 expression profiles and the progression of the tumour - advanced cancers more often showed CK20+/CK7 phenotype, while earlier stag- 
es were mostly CK7(-). CK7 expression was not related to the primary site [4]. In another study performed on 196 patients with colorectal carcinoma a relation between CK20 expression and tumour staging was noted (lower expression in higher stages), as well as with the right-side localisation. CK7 expression was also shown to be related to the presence of metastases. A high proportion of colorectal cancer cases with CK7+ (around $17 \%$ ) and CK20- (up to 19\%), noted in some studies, indicates that the lack of the profile "typical" for this location i.e. CK20+/CK7- in metastases, does not preclude the origin of the primary tumour in the colon [5]. In other studies investigating keratin expression in colorectal cancer, the association of reduced CK8 expression with worse survival was observed (but CK8 expression was not an independent factor associated with TNM staging of the tumour). Reduced CK8 expression is regarded as an indicator of the epithelial-mesenchymal transition process (EMT), leading to more malignant forms of cancer [6]. On the other hand, assessment of colorectal cancer cases with microsatellite instability demonstrated reduced or null expression of CK20. High microsatellite instability (occurring in HNPCC syndrome and in a portion of sporadic cases) identifies a subset of colorectal cancers with abnormally low expression of CK20 [7]. Since CK2O is considered to be one of the markers of colonocyte differentiation, one of the reasons for a reduction in its expression may be tissue de-differentiation in the process of carcinogenesis.

Studies on the prognostic significance of bone marrow micrometastases in patients undergoing "treatment" resection due to gastrointestinal tumours, using flow cytometry analysis of CK18, showed that the presence of $\mathrm{CK} 18(+)$ cells indicates the residual disease and identifies a group of patients potentially eligible for adjuvant therapy. The presence of CK18(+) cells in the bone before surgery showed a clear correlation with worse prognosis - the presence of this population reflects both the transient burst of the cells, the metastatic potential, and the spread of the disease.

Studies investigating the prognosis of patients with epithelial tissue tumours can use simultaneous determination of two different soluble fragments of the CK18 cytokeratin, which will provide information about the total cell death (with M65 antibody total apoptosis and necrosis can be measured) and about apoptosis (with M30 antibody specific to the CK18 fragment cleaved by caspase 3 ). In tumours containing high amounts of CK18 this provides a better insight into the effects of the chemotherapy. In a study on a group of 62 colorectal cancer patients (plus a group of 27 controls), the presence of disseminated tumour cells in the bone marrow was assessed in correlation with quantitative evaluation with M65 and M30 before and after surgery. A positive correlation between a higher number of cancer cells in the bone marrow and higher M65 levels after surgery was found. M65 assay can therefore be used to identify patients with a potentially metastatic course of colorectal cancer [8]. In another study (of 46 patients with colorectal cancer) a significant correlation between M30-labelled fragment and relapses over the 36-month follow-up was found in patients showing no decrease in M30-labelled fragment after surgery. In a small subset of patients undergoing chemotherapy increases in M30 did not correlate with treatment response. Evaluation of M30-labelled fragment concentration could potentially be of use in identifying patients with the disseminated disease (the studies quoted must be regarded as pilot studies due to the small sample size). M30 antibody is also used in evaluating the treatment result, namely differentiating necrosis from apoptosis, in chemotherapy of breast, lung, and prostate cancer.

Evaluation of the cytokeratin expression in cancer research allows some conclusions on the carcinogenesis process: epidemiological, morphological, and molecular data indicate differences between a normal mucosa and the cancer in the right and left side of the colon - in a GeneChip matrix study differences in 58 genes were described. Keratins 8, 19, and 20 and carbonic anhydrases II, IV, and VII showed expression dependent on the localisation - which was lower in the tumours of the left half of the colon; COX-2, on the other hand, had higher expression in this localisation. Differences in CK2O and COX-2 expression were confirmed by immunohistochemical studies. Another interesting finding was different expression of CK7 and MUC6 in tumours related to ulcerative colitis when compared to spontaneous tumours. UC5AC presence was also higher in tumours associated with ulcerative colitis than in sporadic ones, suggesting that "stomach"-type mucins play an important role in the early stages of the colon cancer carcinogenesis. Hence, CK7 and "stomach"-type mucins can be useful in differentiation between cancer developing on the basis of ulcerative colitis and sporadic tumours.

Cytokeratins CK8 and CK18 are a basic set of simple (one-layer) epithelial cytokeratins. They appear early during the embryogenesis of the epithelial cells - as early as the pre-implantation phase. These cytokeratins also seem to be the oldest in the phylogenetic development of the whole keratin family. In the highly specialised epithelial cells, forming parenchymal organs, CK8 and CK18 are the only keratins present - typical examples being hepatocytes, pancreatic follicular cells, renal proximal tubular cells, and some endocrine cells (e.g. pancreatic islets). CK8 and CK18 are 
present in most simple epithelia, often in combination with other keratin pairs, they may also occur focally in stratified epithelia, although typically they are not present in mature keratinocytes. In cancer diagnostics CK8/18 are found in most cancers, with the exception of well-differentiated squamous cell carcinomas, providing evidence of epithelial origin in the case of tumours of unknown origin.

Cytokeratins CK7/CK19 are an additional pair of simple epithelia keratins with a broad tissue distribution. Typically they are found in the epithelia of the excretory ducts, such as bile ducts in the liver and pancreatic ducts. CK19 is the smallest of the keratins, with a broad tissue distribution. With the exception of the epithelia of the mesenchymal organs it is found in most of the simple epithelia, stratified epithelia, and transitional epithelium, as well as in the basal layer of the stratified keratinised epithelium. CK19 is characterised by a lack of helix-like tail domain, which is typical for other keratins. In animal models, the lack of CK19 is compensated for by CK18. In human pathology no diseases related to CK19 defect has been identified thus far. CK19 expression can be used to distinguish HCC from cholangiocarcinoma and metastatic lesions that usually exhibiting strong expression of the keratin (not present in the hepatocytes). In a study of 71 cases of intrahepatic bile duct cancer (cholangiocarcinoma) an association of high levels of CK19 fragments, as evaluated by CYFRA-21-1 test, with tumour progression and poor prognosis after surgery was described. A high concentration of CYFRA 21-1, the presence of micrometastases in lymph nodes, and infiltration of the surgical margin were independent prognostic factors for both relapse and death following post-surgery treatment. When cholangiocarcinoma is to be distinguished from non-malignant lesions in the liver, CK19 evaluated with CYFRA 21-1 showed a discrimination value superior to CEA or CA19.9 evaluated at the same time [9]. In another study 300 hepatocellular carcinoma cases undergoing surgery were evaluated for CK19 and CK10 expression. CK10 expression was found to be associated with poor prognosis and was found to be much higher in the presence of distant metastases, and it influenced the overall survival as well as the duration of relapse-free time. CK19 expression also showed an association with the patients' survival [10]. Measurement of the soluble CK19 fragment is used in monitoring the treatment effects in squamous cell lung carcinoma (CYFRA21-1).

CK7 is a keratin sharing the expression pattern with CK19 but with one, very significant, difference: it shows no, or very weak, expression in the epithelium of the gastric pits, intestinal epithelium, and in the stratified squamous epithelia. The lack of CK7 expression in most cases of colorectal cancer (as opposed to most other adenocarcinomas) provides the basis for the most popular application. The expression is similar in renal clear cell carcinoma and in squamous cell carcinoma of origin other than the cervix. The highest diagnostic power is obtained (in the case of metastatic tumours of unknown origin) when CK7 is evaluated together with CK20. Colon cancer, gastric cancer, and cancers of the transitional epithelium of the urinary tract, as well as skin cancer originating from Merkel cells, show strong expression of CK20. Pancreatic duct cancers and bile duct cancers, and to some extend mucous tumours of the ovaries, show changeable and usually focal expression of CK20. Assessment of CK20-expressing cells in the bone marrow and in the peripheral blood is used in the diagnosis and treatment monitoring of colon, stomach, and pancreatic cancer patients.

CK20 is a typical keratin of the gastrointestinal tract epithelium (stomach, small intestine, and large intestine), urinary tract, and Merkel cells in the skin. Amongst keratins of the simple epithelia it is characterised by the most limited tissue expression. It polymerises primarily with CK8. No clinical implications of CK20 mutations have been identified thus far in human pathology.

CK5 and CK14 are a basic pair of keratins of the stratified, squamous epithelium with expression found predominantly in the basal layer (containing cells with the greatest potential for reproduction, including stem cells), with a gradual reduction of expression paralleling the differentiation degree. CK5/14 keratin pair dysfunction, due to mutations, leads to the reduction of the epithelium stability/resistance (mainly keratinocytes of the basic layer are affected) - which is the molecular basis of EBS (epidermolysis bullosa simplex). CK5/14 pair presence in squamous epithelium is used in the cancer diagnostics - their expression is high in the majority of squamous cell carcinomas and mesotheliomas, while it is missing (or little/focal) in adenocarcinomas, allowing diagnosis particularly in cases of poorly differentiated squamous cell carcinomas, especially in micrometastases in lymph nodes (a typical example being nasopharyngeal carcinoma). Focal expression of CK5 in some cases of endometrial or pancreatic adenocarcinoma is a result of the potential of focal differentiation towards squamous elements. In the case of adenocarcinoma a focal expression of CK17 suggests the tumour originating in the pancreatic ducts.

Unlike keratinising epithelium, striated non-keratinising squamous epithelium of the mucous membranes shows expression of CK4/13 in its superficial layers, which is virtually absent in the epidermis (differentiating element). CK4 is present in the epithelium of the pancreatic ducts (and in the poorly differentiated cases 
of ductal breast carcinoma), which is useful in the diagnosis of tumours originating from these structures.

The use of the keratin expression profiling is not limited to oncology alone. Considering the wide range of keratin function in the epithelial cells, the potential importance of keratin genetic variants in pathology has been recognised. Population studies have shown that mutations of the CK8/18 genes in men are associated with a three-fold increased risk of developing end-stage liver disease (ESLD), regardless of the aetiology. CK8/18 mutations were also found to be related to the risk of progression of chronic hepatitis C. This may be due to mutation-related changes in keratin solubility, filament conformation, and altered susceptibility of keratins to the phosphorylation process. These changes affect not only the function of keratins in maintaining the integrity and structure of the cells and the location and function of intracellular organelles, but also processes involved in protein metabolism, signal transmission, and cytoprotection, especially as far as the anti-apoptotic effect of keratins is concerned. Finally, genetic changes may affect the keratin role in controlling phosphorylation processes and phosphatases activity in the cell (the theory of "phosphate sponge"). Keratins are involved in the formation of Mallory-Denk bodies (MDB), which are morphological determinants of chronic pathological processes in the liver, while CK18 fragments, generated by the action of caspases during apoptosis, are indicators of liver cell damage [11]. These observations are confirmed by animal-model studies - transgenic mice with a human variant of CK8 (G61C), showing CK8 overexpression, are very susceptible to hepatocyte injury, especially when the injury is induced by apoptosis-inducing factors. These mice show a significant decrease in keratin phosphorylation by stress-activated kinases, confirming that keratins act as a kind of "phosphate sponge", modulating intracellular phosphorylation processes in response to kinase-involving stress [12].

Mallory bodies (currently named Mallory-Denk bodies - MDB) represent a histopathological landmark of chronic liver disorders. Initially they were described in alcohol liver disease as irregular cytoplasmatic inclusions of different size, with dense content of mainly CK8, CK18, ubiquitin, and p62 ubiquitin binding protein. The formation of MDB is multi-step and occurs over some years - with the exception of the childhood idiopathic copper metabolism disorder. Typically, MDBs are found in chronic alcohol overuse, chronic poly-metabolic syndrome, and in other metabolic disorders including Wilson's disease. They are also present in chronic cholestasis. Under stress there is an increase in CK8/18 expression (even up to three-fold), with a predominance of keratin CK8 leading to disturbances of balance.
Transglutaminase-2 has a higher affinity towards CK8, compared to CK18. Thus, as a result of transglutaminase- 2 action, keratins are transamidated and have an increased tendency for cross-linking. At the next step, binding to p62 protein takes place, and then ubiquitination with the involvement of the chaperon proteins occurs. Because the protein degradation processes are insufficient, typical cytoplasmatic intrusions are formed. The MDB formation is reversible (requiring removal of the causative factor over a long time) and is characterised by an unusual phenomenon known as "chemical memory" - even though the primary formation MDBs requires a long period of time, after the regression, if the causative agent (i.e. ethanol) re-emerges again, new MDBs are formed very quickly. This phenomenon is not fully explained. It can be associated with the involvement of protein systems that may require a very long time to return to their original state (chaperone involvement is postulated here) $[11,13]$.

In the animal model CK8-negative mice are hypersensitive to liver injury. At the same time, they show abnormalities in the size and function of hepatic cell mitochondria as well as the formation of the anti-mitochondrial antibodies. This observation formed the basis for the evaluation of genetic CK8 variants in the primary liver cirrhosis in humans - with its specific presence of antimitochondrial antibodies. A total of 201 Italian patients with PBC were studied, showing an increased rate of CK8/CK18/CK19 variants in comparison with a healthy population. These variants were also connected with disease progression. The relation between the frequency of the variants and the presence of anti-mitochondrial or anti-nuclear antibodies was not confirmed in this study [14].

Cytokeratin evaluation is also useful in cholelithiasis studies. The CK8/18 expression profile was investigated in patients with cholelithiasis, showing differences between younger (generally lower expression with CK18 predominance) and older patients (higher expression of CK8 related to acute clinical course), suggesting different involvement of these keratins in the pathogenesis of cholelithasis in younger patients [15]. Intense CK7+ expression of diffused type was shown in 25 patients with cholelithiasis with only focal expression of CK20+ in half of the cases [16].

Keratin variants may also be involved in the pathogenesis of chronic pancreatitis. Since overexpression of the human gene KRT8 in a mouse model produces phenotypic changes suggesting chronic pancreatitis (pancreatic mononuclear infiltration, interstitial fibrosis, and acinar cell dysplasia leading to exocrine pancreatic insufficiency), CK8 polymorphism was investigated in patients with chronic pancreatitis from Austria, 
the Czech Republic, Germany, Great Britain, India, Italy, the Netherlands, Romania, Spain, and Switzerland. The frequency of CK8 mutations (G62C and Y54H) was not significantly different in pancreatitis, chronic pancreatitis, pancreatic cancer, and in the control group. In this population mutations of KRT8 were not predisposed to chronic pancreatitis or pancreatic cancer development. The frequency of $\mathrm{G} 62 \mathrm{C}$ variant was investigated in the USA population, again showing no relation to chronic pancreatitis. This study included the following subjects: familiar chronic pancreatitis with PRSS1 mutation (cationic trypsinogen), without PRSS1 mutation, sporadic without mutations, alcoholic, and the control group [17].

In a mouse model without CK8 expression spontaneous colitis in Th2-mediated mechanism develops. This observation was the basis for research on keratin variants in patients with inflammatory bowel disease (IBD). No association of CK8 and CK19 variants with family history of inflammatory bowel disease was described but their potential role in sporadic IBD cannot be ruled out [18], especially as missense mutations of CK8 were identified. In the course of IBD studies expression of CK7 was found in several cases in single cells in the regenerating tissue (CK7 is absent in normal colon mucous membrane). CK7 is also found in all dysplasia cases (with no relation to moderate or severe dysplasia). CK7 expression was also found in cases of minimal intensity staining adenocarcinoma. Thus CK7 may be of potential use in diagnosing dysplasia [19].

Apoptosis process can be assessed with the labelled caspase-cleaved CK18 fragment (M30), especially in relation to total cell death (M65) - it is an important contribution of the methodology of keratin determination to the diagnostics. Unfortunately, these methods are organ unspecific, so they are mainly used in the diagnostics of organs with large mass and high CK18 content (liver pathologies, some cases of cancer).

Non-alcoholic fatty liver disease is one of the biggest health problems in developed societies (in the US it is the most common chronic liver disease). Due to the wide spectrum of clinical manifestations of the disease, one of the major problems is the selection of patients with poor prognosis - pointing out patients with steatohepatitis (NASH). Histopathological evaluation of a liver biopsy is the golden standard of diagnostics - but it is an invasive and risky procedure. One of the key pathomechanisms in steatotic lesions, leading to inflammation, is activation of the apoptosis in the hepatocytes, thus measurement of CK18 caspase-cleaved fragment (M30 test) seems a promising clinical test. It has a predictory value of approximately $99.9 \%$ (positive prediction) and $85.7 \%$ (negative prediction) [20, 21]. Assess- ment of the correlation with histopathology changes demonstrated a very high differentiation rate between healthy subjects and patients with mild changes in the course of a simple steatosis or those with NASH [22]. Patients with simple liver steatosis may still develop NASH with fibrosis progression - all patients should undergo control on a regular basis. The level of CK18 fragments mirrored the disease activity, and changes of CK18 levels correlated with the activity of steatotic changes. Adiponectin, tumour necrosis factor $\alpha$ (TNF- $\alpha$ ), interleukin-6 (IL-6), and leptin levels at baseline and at 36 months were related to the disease progression [23].

In chronic hepatitis $\mathrm{B} \mathrm{HBe}(-)$ patients a positive correlation between the severity of apoptosis, as assessed with the abovementioned method, and the level of transaminases, viral load, and clinical stage (grading) was found. The apoptosis level negatively correlated with the number of platelets, and there was no correlation with the severity of fibrosis and steatosis. Soluble CK18 fragment generated by caspase- 3 seems to be a useful marker for differentiating inactive carriers of HBV and patients with chronic hepatitis B with $\mathrm{HBeAg}(-)$, but it is not helpful in determining the degree of histological liver changes in these patients [24]

When the degree of the histopathological changes was evaluated in patients with chronic hepatitis C a moderate discrimination force was found for caspasecleaved CK18 levels in patients with minimal/mild microscopic lesions versus moderate/severe lesions [22]. A positive correlation with the level of steatosis was also noted in patients with hepatitis $C$. A low response rate to the antiviral regimes is still a significant clinical issue in hepatitis $C$ patients. A positive correlation between the high levels of CK18 fragment, paralleling the activity of apoptosis, and the virus elimination was identified [25].

In a study involving 143 patients with alcohol overuse, serum markers of hepatocyte death (CK18), apoptosis (caspase-cleaved CK18 fragment), and necrosis (CK18-CK18 fragment) were higher in patients with severe fibrosis vs. patients with mild fibrosis. The markers showed a strong correlation with MDBs, ballooning degeneration of the hepatocytes and fibrosis, as well as with hepatic TNF- $\alpha$ and transforming growth factor $\beta$ (TGF- $\beta$ ) levels (measurements in 24 patients). Their role as independent prognostic markers of severe fibrosis has been confirmed in the model comprising alkaline phosphatase (ALP) measurement, bilirubin, international normalized ratio (INR), hialuronian, and apoptosis/necrosis markers. Conclusions: hepatocyte death in alcohol overusing patients can be easily evaluated by measurement of the serum levels of the markers, and it correlates with severe fibrosis [26]. 
Transplant rejection in the graft versus host disease (GvHD) mechanism is the main problem in organ transplantations. As apoptosis in a transplanted organ is a histopathological landmark of GvHD, measurement of the CK18 fragment in the M30 test can be used for non-invasive monitoring of GvHD in liver and intestine transplantations, providing a new diagnostic mechanism for differentiating GvHD symptoms from transplanted organ dysfunctions due to other causes, allowing for early intensification of immunosuppression [27].

Keratin evaluation is useful in many aspects, including the diagnostics and pathophysiology research of the upper GI tract disorders.

In Barrett oesophagus (BE) keratin evaluation is used in the diagnostics as well as in research investigating the pathophysiology of the disorder. Barrett's oesophagus is classically characterised by the presence of goblet cells - alcian blue staining reveals the presence of other cells (columnar blue cells) with a CK7/20 expression pattern similar to goblet cells. In patients with intestinal metaplasia in a normal gastro-oesophageal junction CK7/20 staining pattern allows identification of two groups - with the long BE segment staining patter and with the pattern typical for Helicobacter pylori (Hp) infection [28]. CK8, CK18, and CK20 staining shows the cells with a positive phenotype in both Barrett's oesophagus and in the mucous membrane of the gastric cardia. CK7 expression was found in all cases of Barrett's oesophagus but also in $26 \%$ of cardia bioptates. CK10 and CK13 expression was only found in normal stratified epithelium. CDX-2 was positive in $87.5 \%$ of Barrett's oesophagus cases, while stratified epithelium and normal cardia staining were uniformly negative. CDX-2 with a CK panel can be useful in differentiating the metaplastic epithelium of Barrett's oesophagus and stratified squamous epithelium, while CDX-2 and CK-7 are most meaningful for the distinction between Barrett's oesophagus and normal mucous membrane of the cardia. How to distinguish between a short segment of the Barrett's oesophagus (SSBE) and an ultra-short segment from the intestinal metaplasia of the mucous membrane of the gastric cardia is a separate problem. Distinction between SSBE and intestinal metaplasia of the cardia should not be based on a single diagnostic method alone - it is advisable to simultaneously determine the $C K 7 / 20$ profile, perform mucin staining, and confront the classical histological assessment with the clinical data. The presence of type III metaplasia, reflux disease symptoms (GERD), and CK7/20 expression pattern typical for Barrett's oesophagus suggest SSBE diagnosis, while the confirmed Helicobacter pylori infection, intestinal metaplasia other than type III, and "gastric" pattern of CK7/20 expression suggests metaplasia of the gastric cardia [29]. Sometimes the oesophagus adenocarcinoma evaluation is inconclusive - a study of 62 cancer patients suggests that adenocarcinoma should not always be regarded as related to GERD other possible pathogenetic pathways should also be considered.

From the epidemiological point of view a significant portion of upper GI pathologies are related to Helicobacter pylori infection. In $\mathrm{Hp}(+)$ and $\mathrm{Hp}(-)$ patients with chronic gastritis the pattern of CK8, CK18, and CK19 was compared with healthy tissue. CK20 expression in the antrum was significantly higher in $\mathrm{Hp}(+)$ gastritis patients compared with $\mathrm{Hp}(-)$, and it was also higher than in the normal tissue. Expression in the gastric body was comparable in all groups. Successful $\mathrm{Hp}$ eradication normalised CK20 expression in the antrum within the 6-month follow-up. The K7, K8, CK18/19/20, and Ki67 distribution pattern was found to be significantly different in post-analgesic gastropathy and in Helicobacter pylori gastritis - perhaps reflecting different damage pathways. In adult patients with $\mathrm{Hp}$ infection and cagA+ in the antrum changes in CK7, CK18, CK19, and CK20 expression together with normal CK8 expression can be related to the loosening of the epithelial tight junctions, which is observed in the gastric mucous over the course of the infection (CK 18/19 typical distribution but altered expression intensity in the pit area: CK18 increased expression, CK19 decreased expression in $\mathrm{Hp}(+)$, decreased CK2O in $\mathrm{Hp}(+)$ although high in $\mathrm{Hp}(-)$ gastritis) [30].

The use of the keratin determinations as markers related to the epithelial tissue is extremely versatile: CK18 assay was used in the studies of ischaemic damage pathophysiology in a model of the human colon, keratin was determined in studies on the pathophysiology of irritable bowel syndrome (IBS) in an animal model, keratins have been widely used for the identification/confirmation of the origin of isolated cell lines or experimental models, and keratins were used in an attempt to isolate hepatocyte progenitors (as part of the research on methods allowing for the development of alternative procedures for liver transplantation). Keratins were evaluated in the study on the pathophysiology of $M$ cells, which cover the intestinal lymph follicles and are involved in the transmission and presentation of antigens, and are linked to the primary formation of the aphthous lesions typical for Crohn's disease. Keratins are used in the isolation and labelling of the epithelial cells, in urological models attempting to produce an artificial reservoir in animals with the use of fibrin glue and isolated urothelium cell spray, and finally in research on the pathophysiology of acute pancreatitis, both in the assessment of regeneration stellate cells, based on the necrosectomy material examination, and 
in an animal model. The CK18 (KRT18) gene is a potential vector candidate for gene therapy of cystic fibrosis.

Applying keratin profiling in the diagnostics of many pathologies, including the gastrointestinal tract, is a result of significant progress in basic science. It can serve as an example of the use of cell biology achievements in clinical practice, opening at the same time vast fields for new research.

\section{Conflict of interest}

The authors declare no conflict of interest.

\section{References}

1. Moll R, Divo M, Langbein L. The human keratins: biology and pathology. Histochem Cell Biol 2008; 129: 705-33.

2. Magin TM, Vijayaraj P, Leube RE. Structural and regulatory functions of keratins. Exp Cell Res 2007; 313: 2021-32.

3. Chu P, Wu E, Weiss LM. Cytokeratin 7 and cytokeratin $20 \mathrm{ex}-$ pression in epithelial neoplasms: a survey of 435 cases. Mod Pathol 2000; 13: 962-72.

4. Hernandez BY, Frierson HF, Moskaluk CA, et al. CK2O and CK7 protein expression in colorectal cancer: demonstration of the utility of a population-based tissue microarray. Hum Pathol 2005; 36: 275-81.

5. Bayrak R, Yenidünya S, Haltas H. Cytokeratin 7 and cytokeratin 20 expression in colorectal adenocarcinomas. Pathol Res Pract 2011; 207: 156-60.

6. Knösel T, Emde V, Schlüns K, et al. Cytokeratin profiles identify diagnostic signatures in colorectal cancer using multiplex analysis of tissue microarrays. Cell Oncol 2006; 28: 167-75.

7. McGregor DK, Wu TT, Rashid A, et al. Reduced expression of cytokeratin 20 in colorectal carcinomas with high levels of microsatellite instability. Am J Surg Pathol 2004; 28: 712-8.

8. Ausch C, Buxhofer-Ausch V, Olszewski U, et al. Circulating cytokeratin 18 fragment m65-a potential marker of malignancy in colorectal cancer patients. J Gastrointest Surg 2009; 13 2020-6.

9. Uenishi T, Yamazaki O, Tanaka H, et al. Serum cytokeratin 19 fragment (CYFRA21-1) as a prognostic factor in intrahepatic cholangiocarcinoma. Ann Surg Oncol 2008; 15: 583-9.

10. Yang $X R$, Xu Y, Shi GM, et al. Cytokeratin 10 and cytokeratin 19: predictive markers for poor prognosis in hepatocellular carcinoma patients after curative resection. Clin Cancer Res 2008; 14: 3850-9.

11. Ku NO, Strnad P, Zhong BH, et al. Keratins let liver live: mutations predispose to liver disease and crosslinking generates Mallory-Denk bodies. Hepatology 2007; 46: 1639-49.

12. Ku NO, Omary MB. A disease- and phosphorylation-related nonmechanical function for keratin 8. J Cell Biol 2006; 174 $115-25$.

13. Omary MB, Ku NO, Strnad P, et al. Toward unraveling the complexity of simple epithelial keratins in human disease. J Clin Invest 2009; 119: 1794-805.

14. Zhong B, Strnad P, Selmi C, et al. Keratin variants are overrepresented in primary biliary cirrhosis and associate with disease severity. Hepatology 2009; 50: 546-54.
15. Kasprzak A, Malkowski W, Seraszek A, et al. Cytokeratin 8 and 18 tissue expression In gallbladder muc osa of patients with cholelithiasis. Pol J Pathol 2011; 4: 241-9.

16. Kalekou H, Miliaras D. Cytokeratin 7 and 20 expression in gallbladder carcinoma. Pol J Pathol 2011; 1: 25-30.

17. Schneider A, Lamb J, Barmada MM, et al. Keratin 8 mutations are not associated with familiar sporadic and alcoholic pancreatitis in a population from the United States. Pancreatology 2006; 6: 103-8.

18. Tao GZ, Strnad P, Zhou Q, et al. Analysis of keratin polypeptides 8 and 19n variants in inflammatory bowel disease. Clin Gastroenterol Hepatol 2007; 5: 857-64.

19. Radovic S, Selak I, Babić M, et al. Anti-cytokeartin 7: positive marker for epithelial dysplasia in flat bowel mucosa. Bosn J Basic Med Sci 2004; 4: 24-30.

20. Więckowska A, Zein NN, Yerian LM, et al. In vivo assessment of liver cell apoptosis as a novel biomarker of disease severity in nonalcoholic fatty liver disease; Hepatology 2006; 44: 27-33.

21. Więckowska A, McCullough AJ, Feldstein AE, et al. Noninvasive diagnosis and monitoring of nonalcoholic steatohepatitis: present and future. Hepatology 2007; 46: 582-9.

22. Papatheodoridis GV, Hadziyannis E, Tsochatzis E, et al. Serum apoptotic caspase activity in chronic hepatitis $C$ and nonalcoholic fatty liver disease. J Clin Gastroenterol 2010; 44: 87-95.

23. Wong VW, Wong GL, Choi PC, et al. Disease progression of non-alcoholic fatty liver disease a prospective study with paired liver biopsies at 3 years. Gut 2010; 59: 969-74.

24. Papatheodoridis GV, Hadziyannis E, Tsochatzis E, et al. Serum apoptotic caspase activity as a marker of severity in $\mathrm{HBeAg}$ -negative chronic hepatitis B virus infection. Gut 2008; 57: 500-6.

25. Volkmann X, Cornberg M, Wedemeyer H, et al. Caspase activation is required for antiviral treatment response in chronic hepatitis C virus infection. Hepatology 2006; 43: 1311-6.

26. Lavallard VJ, Bonnafous S, Patouraux S, et al. Serum markers of hepatocyte death and apoptosis are non invasive biomarkers of severe fibrosis in patients with alcoholic liver disease. PLoS One 2011; 6: e17599.

27. Luft T, Conzelmann M, Benner A, et al. Serum cytokeratin-18 fragments as quantitative markers of epithelial apoptosis in liver and intestinal graft-versus-host disease. Blood 2007; 110: 4535-42.

28. Couvelard A, Cauvin JM, Goldfain D, et al. Cytokeratin immunoreactivity of intestinal metaplasia at normal oesophagogastric junction indicates its aetiology. Gut 2001; 49: 761-6.

29. Liu GS, Gong J, Cheng P, et al. Distinction between short-segment Barrett's esophageal and cardiac intestinal metaplasia. World J Gastroenterol 2005; 11: 6360-5.

30. Todorovic V, Sokic-Milutinovic A, Drndarevic N, et al. Expression of cytokeratins in Helicobacter pylori-associated gastritis of adult patients infected with cagA+ strains: an immunohistochemical study. World J Gastroenterol 2006; 12: 1865-73.

Received: 31.10 .2012

Accepted: 7.01.2013 